religiosum vorliegen. Die Schnitte werden am besten mit trockenem Messer aus Material gewonnen, welches in einer Mischung aus gleichen Gewichtsteilen Glyzerin, Spiritus und Wasser eingeweicht war, und unmittelbar vor dem Schneiden oberflächlich mit Filtrierpapier abgetupft ist.

Schliesslich möchte ich noch hervorheben, dass die Figuren 5, 6,7 und 9 sämtlich genau bei 260 facher Vergrösserung mikrophotographisch aufgenommen und dann in genauer Grosse der Photogramme nach den Originalpräparaten gezeichnet sind. Figur 8 ist bei 560 facher Vergrösserung photographiert und danach gezeichnet. Die Grössenverhältnisse sind daher genau bestimmt und unter Berücksichtigung des Vergrösserangsfaktors können exakte Messungen der abgebildeten Objekte vorgenommen werden. Das ist leider bei den von anderen Seiten veröffentlichten Abbildungen nicht immer der Fall. So hat z. B. E. Collin in seiner oben zitierten Mitteilung den Querschnitt eines Fruchtstieles von Illicium verum seiner Angabe nach bei 150 facher Vergrösserung abgebildet. Ein Blick auf die Abbildung zeigt die Unrichtigkeit der Angabe. Die Abbildung besitzt etwa $70 \mathrm{~mm}$ Durchmesser, während die Stiele von Illicium verum nicht unter $1 \mathrm{~mm}$ Durchmesser haben!

Wiesbaden im April 1899.

\title{
Ueber die quantitative Bestimmung des Santonins.
}

\author{
Von J. Katz.
}

(Eingegangen den 19. IV. 1899.)

Bei dem Versuch der Wertbestimmung der homöopathischen Urtinktur von Cina machte sich der Mangel einer wirklich brauchbaren Methode zur quantitativen Bestimmung des Santonins unangenehm bemerkbar, da die bisher bekannten Methoden von Dragendorf $f^{1}$ ), Flückiger und Ehlinger ${ }^{2}$ ) and Thäter ${ }^{8}$ ) in keiner Weise ihrer Aufgabe genügen.

Die Fehler der Dragendorff'schen Methode sind schon von Flückiger hervorgehoben. Diejenigen der Flückiger'schen lassen

1) Dragendorff: Qualitat. und quantitat. Analyse von Pflanzen und Pflanzenteilen 1882, pag. 148.

2) Archiv d. Pharm. 1886, pag. 6.

8) Archiv d. Pharm. 1897, pag. 401-406. 
sich kurz dahin zusammenfassen, dass erstens durch die Auskochung mit alkoholischer Kalkmilch keine vollkommene Extraktion des Santonins herbeigefuhrt wird, dass zweitens eine regelrechte Zersetzung des Calciumsantoninates nicht vorgenommen wird, und drittens die Reinigung des durch Eindampfen der Lösung teilweise freigemachten Santonins mit Kohle nur unvollständig erfolgt. (Die von Thater an dieser Methode geübte Kritik weist mehrere Irrtümer auf; denn santoninsaurer Kalk wird durch Kohlensâure uberhaupt nicht zersetzt und ausserdem wird bei einer Zersetzung von santoninsaurem Kalk Santoninsäure und nicht Santonin gebildet.)

Von der dritten zitierten Methode (von Thäter) behauptet nun zwar der Autor, dass dieselbe das Santonin in nahezu chemischer Reinheit liefere und empfiehlt sie deshalb sogar für die Darstellang des Santonins im Grossen. Abgesehen nun aber davon, dass dieselbe sehr umstăndlich ist, ein Trocknen bei $105^{\circ}$ und die Verwendung von absolutem Aether verlangt (der jedoch bei der vorgeschriebenen 4-5stündigen Extraktion im Soxhlet schwerlich absolut bleiben, vielmehr Wasser anziehen dürfte), konnte ich leider die Thăter'sche Angabe nicht bestätigt finden, trotzdem ich ca. 20 Versuche anstellte und bei denselben die Mengenverhältnisse der einzelnen Reagentien, für die in Thäters Abhandlung jede Angabe fehlt, in der mannigfaltigsten Weise variierte.

Auch bei Kontrollversuchen, die ich mit reinem Santonin vornahm, konnte ich nach Thäters Methode nicht annähernd die angewandte Menge Santonin wiedergewinnen.

Diese Versuche, mit denen ich den Ursachen des Versagens der Methode auf den Grund kommen wollte, waren kurz folgende:

I. 0,2405 Santonin wurden mit 1,0 Aetzkalk und $100 \mathrm{ccm}$ Wasser eine Stunde lang am Rückflusskühler gekocht, darauf filtriert, der Rückstand noch zweimal mit je $50 \mathrm{ccm}$ Wasser ausgekocht und ebenfalls filtriert. Das Filtrat kochte ich mit 75,0 Liquor Aluminii acetici auf, dampfte die stark sauer reagierende Masse auf dem Wasserbade ein, bis sie gallertig wurde, mischte 5,0 Magnesia usta zu, trocknete ganz aus bei $105^{\circ}$ und extrahierte im Soxhl et mit absolutem Aether fünf Standen lang.

Hierbei resultierten $0,0142 \mathrm{~g}$ Santonin, also nur $5,9 \%$ von der angewandten Menge.

II. 0,0880 Santonin und 2,0 Extr. Helenii spirituosum wurden mit 1,0 Actzkalk and $100 \mathrm{ccm}$ Wasser eine Stunde lang gekocht, filtriert und der Rückstand zweimal mit je $50 \mathrm{ccm}$ Wasser ausgekocht. Das Filtrat wurde mit 100,0 Liquor aluminii acetici aufgekocht, eingedickt, mit 5,0 Magnesia usta gemischt, getrocknet und mit Aether extrahiert.

Wiedergefunden wurden 0,0100 Santonin, also $11,6 \%$ von der angewandten Menge. 
III. Da nun beim Versetzen mit Magnesia usta die Masse jedesmal eine alkalische Reaktion annahm, machte ich einen Versuch, bei dem ich statt der Magnesia 10,0 Kieselguhr anwandte, um die Masse voluminöser zu machen.

Von 0,4940 angewandten Santonins erhielt ich dabei wieder 0,2814, also $56,9 \%$.

Hieraus erhellt, dass die Lösung des Santonins in rein wăsseriger Kalkmilıh so langsam vor sich geht, dass allein dadurch schon Verlust entsteht.

Ich machte daher einen Versuch mit Kalkmilch unter Zusatz von Alkohol und zwar in folgenden Mengenverhältnișsen:

IV. 0,3377 Santonin, 2,0 Aetzkalk, $50 \mathrm{ccm}$ Wasser, $50 \mathrm{ccm}$ Alkohol von 96\%. Darauf wird noch zweimal mit je $50 \mathrm{ccm}$ Wasser ausgekocht, das Filtrat mit 25,0 Liquor aluminii acetici aufgekocht, wodurch es stark sauer reagiert, dann auf dem Wasserbade eingedickt, mit 5,0 Magnesia usta und 5,0 Kieselgahr gemischt und, nach dem Trocknen bei $105^{\circ}$, mit Aether im Soxhlet 5 Stunden lang extrahiert.

Hierbei wurden wiedergefunden 0,0934 Santonin $=28,9 \%$.

Da also auch hierbei nur der kleinere Teil des Santonins wiedergewonnen wurde, musste ich eine Bindung der Santoninsäure durch die Magnesia annehmen, was durch einen weiteren Versuch, in dem ich die letztere durch Zinkoxyd ersetzte, bestätigt wurde. Die hierbei angewandten Mengenverhăltnisse waren folgende:

V. 0,2650 Santonin, 1,0 Aetzkalk, $100 \mathrm{ccm}$ Alkohol von $50 \%$, zweimal $50 \mathrm{ccm}$ Wasser, 25,0 Liquor aluminii acetici, 5,0 Zinkoxyd und 10,0 Kieselguhr.

Hierbei wurden wiedererbalten 0,2530 Santonin $=95,5 \%$.

Allein der Kolbeninhalt roch nach dem Abdampfen und vor dem Trocknen sehr stark nach Essigsăure und da nun, wie schon Thäter angiebt, die geringste Menge freier Essigsäure grosse Mengen von Harz und Farbstoff in Lösung bringt, so lässt sich das Zinkoxyd eben wegen der leichten Zersetzbarkeit des Zinkacetates hier nicht zum Neutralisieren der Essigsäure verwenden.

Mit diesen fünf Versuchen ist wohl deutlich bewiesen, dass der Thäter'schen Methode zwei Fehler anhaften:

1. Es ist durch Kochen von Santonin mit wässeriger Kalkmilch auch während einer Stunde keine vollständige Salzbildung zu erreichen,

2. Es wird durch die zugesetzte Magnesia usta der grösste Teil der freigemachten Santoninsäure (denn diese wird bei Abwesenheit von starken Säuren erst sehr langsam in Santonin umgewandelt) wieder als Magnesiumsalz gebunden.

Nun steht zwar dem die Behauptung Thäter's entgegen: "dass selbst beim Kochen von Magnesiumoxyd mit Santonin in reichlichen Mengen Wasser nur äusserst schwierig eine Salzbildung zu stande 
kam." Allein ich fand bei einer Nachprüfung diese Behauptung nicht bestatigt, wie folgender Versuch beweist:

0,3516 Santonin wurden mit 2,0 Magnesia usta und $50 \mathrm{ccm}$ Wasser eine halbe Stunde lang am Rückflusskühler gekocht, die Flüssigkeit darauf eingedampft und der Rückstand im Soxhlet mit Aether erschöpft. Hierbei warden 0,1254 Santonin wiedergewonnen. Es waren also $0,3516-0,1254=$ 0,2262 oder 64,3\% an Magnesia gebunden.

Während nun bei diesem Versuche das Santonin krystallinisch, also für die Magnesia ziemlich schwer angreifbar, vorlag, gestalten sich die Verhältnisse für die Bildung von Magnesiumsantoninat bei der Thäter'schen Methode noch günstiger, da hier das Santonin in der Flüssigkeit bis zuletzt durch die gegenwärtige Essigsäure gelöst bleibt und teilweise sogar noch in Form von Santoninsäure vorhanden ist.

Was nun die Resultate der schon oben erwähnten Versuche, mit Hilfe der Thäter'schen Methode das Santonin in den Flores Cinae zu bestimmen, anlangt, so schwankten dieselben bei Anwendung von 10,0 Zittwersamen zwischen 0,015 and 0,071 oder 0,15 und $0,71 \%$. Dies Santonin war ausserdem nichts weniger als rein, sondern bestand grösstenteils aus einer harzartigen Masse, in der kleine Santoninkrystalle eingebettet lagen.

Als ich die Methode etwas abänderte und statt der Magnesia usta Magnesia carbonica anwandte, erhielt ich 0,3120 und 0,3032 Rückstand, der aber fast ausschliesslich aus Harz bestand, ebenso auch, als ich, wie in Versuch $V$, statt der Magnesia Zinkoxyd nahm, und zwar resultierten hierbei 0,2474 einer Masse, die, wie nicht anders zu erwarten war, nur oder fast nur Harz enthielt.

Auch verschiedene andere Versuche, die ich anstellte, am die Thätersche Methode so abzuändern, dass damit eine Bestimmung des Santonins möglich wäre, misslangen vollständig.

So z. B. zersetzte ich die Lösung von santoninsaurem Kalk mit Schwefelsäure in der Wärme und dampfte nach Zusatz einer gentigenden Menge Aluminiumsulfat und Aetzammon bis zur alkalischen Reaktion, sowie von Kiese]guhr, zur Trockne und extrahierte mit Aether. Dieser Versuch grundete sich darauf, dass Santonin und Ammoniak keine Verbindung eingehen und das Santonin nach dem Verdunsten des Ammoniaks in unveränderter Form zurlickbleibt. Allein auch hierbei wurden grosse Mengen von Harz mitgelöst.

Auch habe ich versucht, die Calciumsantoninatlösung unter Einleitung eines konstanten Kohlensäurestromes durch Kochen zu zersetzen und das Santonin darauf nach dem Eindampfen mit Aether zu extrahieren, da ja bekanntlich die Lơsungen der santoninsauren Erdalkalisalze durch Kochen eine teilweise Zersetzung erleiden, die ich 
durch die Einwirkung der Kohlensäure zu vervollstăndigen hoffte. Hierbei blieb jedoch die Zersetzung selbst nach einstündigem Kochen noch unvollständig, so dass ich in zwei Proben von Flores Cinae, die nach der spater zu beschreibenden Methode 2,479 und 2,948\% Santonin enthielten, nur 1,814 resp. 1,314\% Santonin fand, das noch daza stark mit Harz verunreinigt war.

Ich suchte daher eine neue exakte Methode aufzufinden, was mir denn auch endlich gelungen ist, und machte hierfür folgende orientierende Versuche:

Da sich, wie oben gezeigt wurde, Kalkmilch nicht besonders gut zum Lösen des Santonins eignet, versuchte ich es mit dem stärkeren und auch besser kontrollierbaren Barytwasser.

Ich kochte daher 0,5914 Santonin mit 100,0 Barytwasser am Rückflusskühler, wobei schon nach 5 Minuten völlige Lösung eintrat, und setzte das Kochen im Ganzen 10 Minuten fort. Nach dem Erkalten schüttelte ich die Flüssigkeit mit Chloroform aus und verdampfte das Chloroform. Da hierbei kein wägbarer Rückstand hinterblieb, so ist damit der Beweis erbracht, dass durch Barytwasser eine sehr schnelle und vollständige Salzbildung des Santonins veranlasst wird.

Weiter untersuchte ich dann, ob durch Kohlensäure eine Zersetzang des gebildeten santoninsauren Baryts bewirkt werden kann. Ich leitete also in die Lơsung solange einen langsamen Strom von Kohlensäure ein, bis die alkalische Reaktion vollständig verschwunden war und durch die vorïbergehende Rötung von blauem Lackmuspapier ein Ueberschuss von Kohlensảure angezeigt wurde. Darauf filtrierte ich das Baryumkarbonat ab und extrahierte den mit Wasser ausgewaschenen und dann getrockneten Niederschlag im Soxhlet mit Aether. Hierbei erhielt ich $0,0017=0,29 \%$ des angewandten Santonins.

Das Filtrat vom Baryumkarbonatniederschlag wurde einmal anfgekocht und auf dem Wasserbade bis auf $50 \mathrm{ccm}$ eingedampft. Hierbei bildeten sich Ausscheidungen, grösstenteils aus Baryumkarbonat bestehend, die nach dem Erkalten abfiltriert und ausgewaschen wurden. Nach dem Trocknen wurden sie ebenfalls im S oxhlet mit Aether extrahiert und lieferten 0,1270 Santonin $=21,5 \%$ des angewandten.

Das zuletzt verbleibende Filtrat wurde mit Salzsäure zersetzt, einmal aufgekocht (um die freigemachte Santoninsäure in Santonin zu verwandeln) und nach dem Erkalten dreimal mit Chloroform ausgeschüttelt. Nach dem Verdunsten des letzteren verblieben 0,4610 Santonin.

Es warden also wiedergefunden: $(0,4610+0,1270+0,0017)=0,5897 \mathrm{~g}$ Santonin, angewandt 0,5914 g, Verlust also 0,0017 g Santonin.

Es erhellt aus diesem Versuch, dass Baryumsantoninat in der Kälte durch Kohlensäure nur in einem wohl zu vernachlässigenden Grade zersetzt wird, dass dagegen beim Kochen einer nentralen Baryumsantoninatlosung eine teilweise Zersetzung durch Hydrolyse herbeigeführt wird. 
Weiter studierte ich die Löslichkeit von Santonin in Wasser und in verdünntem Alkohol von verschiedener Stärke:

1. 3,0 Santonin wurden mit $500 \mathrm{ccm}$ Wasser eine Viertelstunde lang gekocht und kochend heiss filtriert. Das Filtrat betrug $450 \mathrm{~g}$. Nach 24 Stunden wurde rom ausgeschiedenen Santonin abfiltriert und letzteres getrocknet and gewogen. Es betrug $1,2210 \mathrm{~g}$. Vom Filtrat wurden $50 \mathrm{ccm}$ verdampft und hinterliessen nach dem Trocknen 0,0130 g Santonin.

(Bei einer zur Kontrolle vorgenommenen Titration mit $n / 10$ Kalilauge in der Kochhitze wurden für $50 \mathrm{ccm}$ Filtrat $0,5 \mathrm{ccm} \mathrm{n} / 10$ Kalilauge $=0,0123$ Santonin verbraucht.)

Die $450 \mathrm{~g}$ Filtrat enthielten also $9 \times 0,013=0,117$ Santonin gelöst und im Ganzen waren durchs Kochen $(0,1170+1,2210)=1,3880$ Santonin in 450,0 Wasser gelöst, d. h. $0,297 \%$ oder 1 Teil Santonin in 337 Teilen Wasser.

2. 0,3406 Santonin wurden mit ca. 50,0 Alkohol von $30 \%$ am Rückflusskühler gekocht, wobei sehr schnell völlige Lösung eintrat. Nach dem Erkalten und Stehenlassen während 24 Stunden wurde filtriert und $46,5 \mathrm{~g}$ Filtrat erhalten, die beim Eindampfen 0,0600 Santonin $=0,129 \%$ hinterliessen.

3. 0,2906 Santonin und ca. 65,0 Alkohol von $15 \%$ warden am Rückflusskühler gekocht. Nach ca. 5 Minuten war völlige Lösung eingetreten. Nach 24 stündigem Stehen in der Kälte worde filtriert und 60,0 g Filtrat erhalten, das 0,0325 Santonin $=0,0542 \%$ enthielt.

4. 1,0 Santonin wurde mit $50 \mathrm{ccm}$ Alkohol von $15 \% 10$ Minuten lang am Rückflusskühler gekocht und kochend heiss filtriert. Das Filtrat wog $45,0 \mathrm{~g}$. Nach 24 stündigem Stehenlassen wurde das ausgeschiedene Santonin abfiltriert, getrocknet und gewogen. Fs betrug 0,3982 g. Das Filtrat hinterliess nach dem Eindampfen noch 0,0302 Santonin $=0,065 \%$. Im Ganzen wurden also von 45,0 kochendem Alkohol von $15 \%$ 0,4284 Santonin $=0,951 \%$ gelöst.

5. 0,3224 Santonin wurden mit $50 \mathrm{~g}$ Alkohol von $10 \% 10$ Minuten lang gekocht, wodurch jedoch keine völlige Lösung erzielt werden konnte. Nach dem Filtrieren bei Kochhitze resultierten $47 \mathrm{~g}$ Filtrat, die 24 Stunden bei seite gestellt wurden. Darauf wurde das ausgeschiedene Santonin abfiltriert, getrocknet and gewogen, es betrug $0,2184 \mathrm{~g}=0,465 \%$. Im Filtrat waren noch 0,0180 Santonin $=0,038 \underset{10}{\alpha}$ vorhanden.

Aus diesen Versuchen folgt, dass der $15 \%$ ige Alkohol für meine Zwecke sich am besten eignet, da die ans 10,0 Flores Cinae zu er. wartende Santoninmenge bequem von ca. 50,0 Alkohol von $15 \%$ gelöst wird und andererseits sich in diesem Alkohol nur sehr wenig Santoninharz beim Kochen löst, das sich beim Erkalten in sehr feinen milchigen Tröpfchen ausscheidet, die glatt durchs Filter gehen, wie ein Versuch lehrte.

Auf Grund vorstehender Versuche habe ich nun folgende Methode für die quantitative Bestimmung des Santonins in den Flores Cinae ausgearbeitet: 
10,0 grob gepulverte Flores Cinae werden im Soxhletapparat 2 Stunden lang mit Aether (Ph. G. III) extrahiert und der Aether abdestilliert. Es hinterbleiben ca. 1,5-2,0 g eines dunkelgrünen, harzigen Extraktes. Dasselbe wird mit einer Lösung von 5,0 krystallisiertem Barythydrat in $100 \mathrm{ccm}$ Wasser $1 / 4$ bis 1/2 Stunde am Rückflusskühler gekocht. Man lässt erkalten und sättigt die kalte Flüssigkeit ohne vorher zu filtrieren mit Kohlensäure, bis eingetauchtes blanes Lackmuspapier gerötet wird. Darauf wird ohne Verzug vom Baryumkarbonatniederschlag abfiltriert, am besten auf einem Saugfilter, und zweimal mit je ca. $20 \mathrm{ccm}$ Wasser nachgewaschen. Man erhält eine blass weingelb gefärbte Flüssigkeit, die man auf dem Wasserbade bis auf ungefähr $20 \mathrm{ccm}$ eindampft. Darauf setzt man $10 \mathrm{ccm}$ verdünnte Salzsäure $(12,5 \% \mathrm{HCl}) \mathrm{zu}_{1}$ lässt noch zwei Minuten (nicht länger) auf dem Wasserbade stehen und giebt die saure Flüssigkeit nach dem Erkalten in einen Scheidetrichter. Die in der Schale zurückbleibenden Santoninkrystalle löst man in ca. $20 \mathrm{~cm}$ Chloroform, bringt diese Lösung ebenfalls in den Scheidetrichter und schüttelt gut durch. Nach dem Absetzen filtriert man die Chloroformlösung durch ein mit Chloroform befeuchtetes Filter und wäscht Schale, Scheidetrichter und Filter zweimal mit je $20 \mathrm{ccm}$ Chloroform nach.

Das Chloroform wird abdestilliert und der Rückstand mit $50 \mathrm{ccm}$ Alkohol von $15 \%$ zehn Minuten lang am Rücktlusskühler gekocht. Man filtriert heiss in ein genau gewogenes Kölbchen und wäscht Kolben and Filter zweimal mit je $10 \mathrm{ccm}$ kochendem Alkohol von $15 \%$ aus. Man bedeckt nun das Kölbchen mit einem Uhrglase und stellt es 24 Stunden in der Kälte bei seite.

Nach dieser Zeit wägt man das Kölbchen mit Inhalt, filtriert durch ein gewogenes Filter ${ }^{1}$ ) von $9 \mathrm{~cm}$ Durchmesser (ohne Rücksicht darauf, dass das Filtrat von feinen Harztröpfchen milchig getrübt ist) und wäscht Kölbchen und Filter mit $10 \mathrm{ccm}$ Alkohol von $15 \%$ (der bei der später anzubringenden Korrektur nicht mit in Anrechnung kommt) einmal aus.

Darauf trocknet man das Filter in dem Kölbchen und wägt.

$\mathrm{Zu}$ dem so gefundenen krystallisierten und schwach gelblich gefärbten Santonin ist noch als Korrektur das im Alkohol gelöst gebliebene Santonin $\mathbf{z a}$ addieren und $\mathrm{zwar}$ sind (unter genauer Innehaltung obiger Zeit- etc. Angaben) für je $10 \mathrm{~g}$ Filtrat 0,006 Santonin in Anrechnung zu bringen.

Trotzdem die alkoholische Lösung nur sehr schwach gefärbt ist, enthælt sie doch noch so viel Harz gelöst, dass ein direktes Eindampfen und Wägen nicht angängig ist.

Es wurden beispielsweise in $10 \mathrm{~g}$ einer Probe Zittwersamen nach obiger Methode durch Auskrystallisieren (inklusive Korrektur) 0,316 Santonin gefunden, während durch einfaches Eindampfen 0,545 Santonin erhalten wurden, also 0,229 $\mathrm{g}$ mehr.

1) Zu dieser Filtration nimmt man zweckmässig ein sehr schnell filtrierendes, nicht za dichtes Papier, da ein Durchlaufen der grossen Santoninkrystalle nicht zu befürchten ist. 
Bei einer vorgenommenen Titration verbrauchten die $0,229 \mathrm{~g}$ Harz 7,9 ccm n/10 Kalilauge, woraus folgt, dass die Harze fast dasselbe Aequivalentgewicht besitzen wie Santonin.

Ausser dieser Bestimmung durch Wägung habe ich auch eine titrimetrische Methode auszuarbeiten versucht, die darauf basiert, dass die freien Harzsäuren, welche die Verunreinigung des Santonins ausmachen, in alkoholischer Lösung bereits in der Kälte mit Kalilauge reagieren, während Santonin erst bei ca. $80-90^{\circ}$ mit Kalilange santoninsaures Kali bildet.

Hierbei war aber vor allem der Umstand sehr störend, dass Glas in der Kochhitze von Kalilauge ganz bedeutend angegriffen wird and man daher immer einen blinden Versuch mit demselben Kolben nebenher machen muss.

Beispielsweise verbrauchten $15 \mathrm{~cm} \mathrm{n} / 10$ Kalilauge mit $30 \mathrm{ccm}$ $96 \%$ igem Alkohol einmal zum Aufkochen erhitzt bei Verwendung von Jenenser Glas nur noch 14,35 ccm n/10 Salzsäure und bei gewöhnlichem Glas $14,50 \mathrm{ccm} \mathrm{n} / 10$ Salzsäure.

Auch bei Anwendung von Porzellangefässen muss man eine Korrektur anbringen, da dieselben in der Kochhitze wohl dem Angriff von Săuren, nicht aber dem von Alkalien widerstehen.

Ich kochte z. B. in einem mit Uhrglas bedeckten Kochbecher aus Berliner Porzellan (das doch die beste Glasur besitzt) $15 \mathrm{ccm}$ n/10 Kalilauge mit $50 \mathrm{ccm}$ Alkohol von $45 \%$ einmal auf und verbrauchte darauf nach Zusatz von Phenolphtalein nur noch $14,4 \mathrm{ccm}$ $\mathrm{n} / 10$ Salzsăure, also waren $0,6 \mathrm{ccm} \mathrm{n} / 10$ Kalilauge durch die Kieselsäure der Porzellanglasur gebunden.

Dass die Harzsäuren bereits in der Kalte vollstandig in wenigen Minuten verseift werden, wurde durch folgenden Versuch konstatiert: 0,0640 völlig santoninfreies Cinaharz wurden durch Erwärmen in $50 \mathrm{ccm}$ Alkohol von $45 \%$ gelöst. Nach dem Erkalten wurden drei Tropfen Phenolphtalein zugesetzt und mit $\mathrm{n} / 10 \mathrm{Kalilauge} \mathrm{titriert.} \mathrm{Es} \mathrm{wurden}$ verbraucht $2,2 \mathrm{ccm} \mathrm{n} / 10$ Kalilauge. Darauf wurden weitere $2 \mathrm{ccm}$ n/10 Kalilange zugesetzt und einmal aufgekocht. Beim Zurücktitrieren wurden nur $1,8 \mathrm{ccm} \mathrm{n} / 10$ Salzsäure verbraucht. Die $0,2 \mathrm{ccm} \mathrm{n} / 10 \mathrm{Kali}-$ lange, welche durch das Kochen verbraucht waren, müssen als vom Glase gebunden angesehen werden.

Für die Titration des Santonins in der nach der oben angegebenen Methode erhaltenen Lösung in $15 \%$ igen Alkohol wird die letztere eingedampft und der Rückstand in $20-30 \mathrm{ccm}$ absolutem Alkohol gelöst. Man versetzt alsdann nach Zugabe von drei Tropfen Phenolphtalein solange mit n/10 Kalilauge, bis eine deutliche Rosafärbung der Flüssigkeit eintritt, die zehn Minuten lang Bestand hält. Dann giebt man 
$20 \mathrm{ccm}$ n/10 Kalilauge zu, erbitzt einmal zum Aufkochen, setzt $50 \mathrm{ccm}$ kaltes Wasser za, wodurch die Farbe des Phenolphtaleins intensiver wird und titriert sofort mit $\mathbf{n} / \mathbf{1 0}$ Salzsäure bis zur Gelbfärbang zuruck.

Durch einen blinden Versuch ermittelt man dann tür denselben Kolben diejenige Menge $\mathrm{n} / 10$ Kalilange, die unter denselben Bedingungen vom Glase gebunden wird. Die nach Abzug dieser letzteren Menge, sowie der verbrauchten $\mathrm{ccm} n / 10$ Salzsäure von $20 \mathrm{ccm}$ verbleibende Anzahl ccm n/10 Kalilauge mit 0,0246 multipliziert, ergiebt die vorhandene Menge Santonin.

Allein abgesehen davon, dass infolge der gelben Farbe der Flüssigkeit (herrübrend von den Harzen) der Endpunkt der Reaktion nicht sehr scharf zu erkennen ist, wird die Menge des Santonins stets etwas zu hoch gefunden werden.

So wurden durch Wägang in einer Probe $3,16 \%$ Santonin und durch Titration einmal 3,21\% und ein anderes Mal 3,38\% Santonin gefunden.

Durch einen Versuch hatte ich mich vorher von der Möglichkeit, Santonin in alkoholischer Lösung zu titrieren, überzeugt. Es warden 0,3080 Santonin in 50,0 Alkohol von $45 \%$ gelöst und nach Zusatz von 3 Tropfen Phenolphtalein $0,1 \mathrm{ccm} \mathrm{n} / 10 \mathrm{Kalilauge}$ zugefügt. Die Rotfärbung war nach 10 Minuten noch deutlich sichtbar. Dann setzte ich $20,4 \mathrm{ccm} \mathrm{n} / 10$ Kalilauge $\mathrm{zu}$, kocbte einmal auf und titrierte mit n/10 Salzsäure zurück. Es wurden $7,3 \mathrm{ccm}$ n/10 Salzsäure verbraucht, ab von $20,4 \mathrm{ccm}$ bleiben $13,1 \mathrm{ccm} \mathrm{n} / 10 \mathrm{Kalilauge,} \mathrm{ab} \mathrm{ausserdem} 0,5 \mathrm{ccm}$ $\mathrm{n} / 10$ Kalilauge, die bei einem Kontrollversuch vom Glase gebunden wurden, bleiben für Santonin $12,6 \mathrm{ccm} n / 10$ Kalilauge $=0,3100$ Santonin. Es wurden also zuviel gefunden 0,0020 Santonin $=0,65 \%$.

Durch eine solche Titration lässt sich andererseits sehr gut die Reinheit des bei der oben ausgeführten gewichtsanalytischen Methode erhaltenen Santonins ermitteln.

Es wurden beispielsweise 0,2625 der bei einer Analyse gewonnenen Santoninkrystalle in $50 \mathrm{ccm}$ Alkohol von $45 \%$ gelöst und in der Kålte nach Zusatz von Phenolphtalein mit $\mathbf{n} / 10 \mathrm{Kalilange} \mathrm{bis} \mathrm{zur} \mathrm{bleibenden}$ Rötung versetzt. Darauf wurde mit $15,37 \mathrm{ccm} \mathrm{n} / 10 \mathrm{Kalilauge} \mathrm{einmal}$ aufgekocht und mit $\mathbf{n} / 10$ Salzsäure zurücktitriert. Verbraucht wurden $4,55 \mathrm{ccm} n / 10$ Salzsäure, die nebst $0,5 \mathrm{ccm}$ vom Glase gebundener n/10 Kalilauge von den $15,37 \mathrm{ccm}$ abzuziehen sind.

Es verbleiben alsdann $10,32 \mathrm{ccm} \mathrm{n} / 10 \mathrm{Kalilange,} \mathrm{entsprechend}$ 0,2540 Santonin. Mithin enthielt das Santonin nur 3,2\% Verunreinigungen. 
Um nun die oben beschriebene gewichtsanalytische Methode auf ihre Brauchbarkeit zu prufen, kochte ich 0,2770 Santonin mit 5,0 Barythydrat und $100 \mathrm{ccm}$ Wasser 15 Minuten lang am Rückflusskühler, săttigte nach dem Erkalten mit Kohlensäure, filtrierte, dampfte ein, zersetzte in der Warme mit Salzsäure und nahm in der oben beschriebenen Weise mit Cloroform auf. Nach dem Abdestillieren des Chloroforms löste ich den Rückstand in $15 \%$ igem Alkohol durch Kochen und filtrierte nach 24 Stunden. Ich erhielt so 0,2260 Santonin in Krystallen und noch 0,0380 Santonin durch Abdampfen der Lösung.

$$
\begin{aligned}
& \text { Angewandt } \quad 0,2770 \text { Santonin }
\end{aligned}
$$

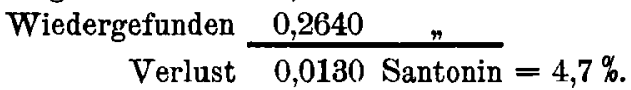

Bei einem $z$ weiten Versuch fand ich von 0,2862 angewandtem Santonin sogar 0,2396 krystallisiertes und 0,0438 gelöst gebliebenes Santonin wieder, also insgesamt 0,2834 .

Verlust hierbei 0,0028 Santonin $=1,0 \%$.

Ich habe nun nach dieser Methode zehn verschiedene Handelsmarken von Flores Cinae untersucht und dabei folgende Werte erhalten:

Santoningehalt der Flores Cinae.

\begin{tabular}{c|c|c|c|c|c|c|c|c|c|c}
\hline \hline Marke & I & II & III & IV & V & VI & VII & VIII & IX & X \\
\hline \hline Versuch I & 2,454 & 2,790 & 2,933 & 2,109 & 2,818 & 1,183 & 3,146 & 2,170 & 2,683 & 1,812 \\
\hline Versuch II & 2,505 & 2,770 & 2,963 & 2,216 & 2,888 & 1,240 & 3,172 & 2,136 & 2,744 & 1,766 \\
\hline Mittel & 2,479 & 2,780 & 2,948 & 2,162 & 2,851 & 1,211 & 3,159 & 2,153 & 2,714 & 1,789
\end{tabular}

Der Santoningehalt der Cinablüten schwankte also bei diesen zehn Proben zwischen $1,211 \%$ und 3,159\% und betrug im Mittel 2,424\%.

Auf gleiche Weise untersuchte ich sodann die aus obigen Handelsmarken durch Perkolation mit Alkohol von 90\% im Verhältnis 1:5 hergestellten homöopathischen Urtinkturen.

Hierbei ist natürlich eine Extraktion mit Aether unnötig, man dampft vielmehr in einem Kolben den Alkohol aus $50 \mathrm{ccm}$ oder aus $50 \mathrm{~g}$ der Tinktur ab und behandelt den Rückstand direkt mit Barytwasser in der oben angegebenen Weise. 
Die hierbei erhaltenen Werte sind folgende:

Santoningehalt von Cinatinkturen (1:5 Alkohol von $90 \%$ ).

\begin{tabular}{c|c|c|c|c|c|c|c|c|c|c}
\hline \hline Marke & I & II & III & IV & V & VI & VII & VIII & IX & X \\
\hline \hline Versuch I & 0,473 & 0,483 & 0,587 & 0,421 & 0,491 & 0,2135 & 0,628 & 0,410 & 0,512 & 0,193 \\
\hline Versuch II & 0,494 & 0,499 & 0,590 & 0,410 & 0,481 & 0,2105 & 0,628 & 0,406 & 0,505 & 0,195 \\
\hline Mittel & 0,484 & 0,491 & 0,589 & 0,416 & 0,486 & 0,212 & 0,628 & 0,408 & 0,508 & 0,194
\end{tabular}

Aehnlich wurde eine quantitative Bestimmung des Santonins in toxikologischen Fällen zu geschehen haben. Man extrahiert die betreffende Masse mit Chloroform (eventuell nach dem. Ansäuern mit Salzsänre) und behandelt das Chloroformextrakt wie oben angegeben mit Barytwasser etc.

Von pharmazeutischen Präparaten, in denen quantitative Santoninbestimmungen auszuführen wären, kommen ausser den schon besprochenen homöopathischen Urtinkturen vor allem noch die Santoninzeltchen in Betracht.

Während man nun aus den mit Zuckerschaummasse hergestellten Zeltchen das Santonin durch einfaches Extrahieren des gepulverten Präparates mit Chloroform in genligender Reinheit isolieren kann, ist bei den Santoninschokoladepastillen das oben beschriebene Verfahren in etwas vereinfachter Form einzuschlagen.

Man kocht drei bis vier der betreffenden, einzeln genau gewogenen, Pastillen mit 5,0 Barythydrat und $100 \mathrm{ccm}$ Wasser eine Viertelstunde am Rückflusskühler und sättigt die Flüssigkeit nach dem Erkalten mit Kohlensäure. Man filtriert, wäscht den Rückstand mit Wasser aus und dampft das bräunliche Filtrat auf ca. $10 \mathrm{ccm}$ ein. Nach dem Zersetzen der Flüssigkeit in der Wärme mit $10 \mathrm{ccm}$ verdünnter Salzäure lässt sich das Santonin durch dreimaliges Ausschütteln mit Chloroform in nahezu vollkommener Reinheit gewinnen, so dass man die Chloroformlösung nur einzudampfen braucht und die letzten Reste des Chloroforms unter Zusatz von wenigen ccm Aether (Alkohol ist hier nicht zu empfehlen) wegzukochen, um nahezu weisse Santoninkrystalle zu erhalten, die einfach gewogen werden.

Sollte dennoch vielleicht durch nicht ganz klares Filtrieren der Baryumsantoninatlösung eine Verunreinigung mit Spuren Fettsäuren stattgefunden haben, so kann man die Santoninkryställchen hiervon 
durch einmaliges Auf kochen mit 10-20 ccm Petroläther und nachfolgendes Filtrieren nach dem Erkalten befreien, da Santonin in kaltem Petroläther fast unlöslich ist, wie folgender Versuch beweist: 1,0 Santonin wurden mit Petroläther eine Stunde lang am Rückflusskühler gekocht, erkalten gelassen und nach dreistündigem Stehen in der Kälte filtriert. Das Filtrat betrug $40,9 \mathrm{~g}$ und hinterliess nach dem Eindampfen 0,0046 Santonin.

Es waren also nur $0,0113 \%$ Santonin im kalten Petroläther gelöst geblieben oder 1 Teil Santonin auf 8900 Teile Petroläther.

Die Brauchbarkeit der abgekürzten Methode für den angegebenen Zweck erprobte ich durch folgende Versuche:

I. 0,1440 Santonin und 5,0 Schokolade (rein Kakao und Zucker) wurden, wie vorgeschrieben, mit Barytwasser etc. bekandelt. Wiedergefunden wurden 0,1432 Santonin. Verlust also nur 0,0008 Santonin.

II. Bei Anwendung von 0,1580 Santonin und 6,0 Schokolade resultierten 0,1554 Santonin. Verlust $=0,0026$ Santonin.

Leipzig-Reudnitz im April 1899.

Chemisches Laboratorium von Dr. W. Schwabe.

\title{
Arbeiten aus dem pharmazeutischen Institute der Universităt Bern.
}

\section{Untersuchungen tiber die Sekrete.}

\author{
Von A. Tschirch. \\ 30. Ueber das Umbelliferen-Opopanax. \\ Von A. Knitl.
}

(Eingegangen den 2. V. 1899.)

Im pharmazeutischen Institut der Universität Bern wurde 1895 das unter dem Namen Opopanax ${ }^{1}$ ) jetzt im Handel befindliche, aus Syrien stammende, und zu Zwecken der Fabrikation von Parfums verwendete aromatische Produkt einer chemischen und botanischmikroskopischen Untersuchung von Tschirch und Baur ${ }^{2}$ ) unterworfen.

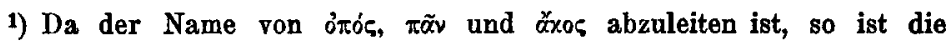
Schreibweise Opopanax richtiger als Opoponax.

$\mathrm{T}$.

8) Archiv d. Pharm. 1895. 